\title{
From ferromagnetism to spin-density wave: Magnetism in the two channel periodic Anderson model
}

\author{
Frithjof B. Anders \\ Institut für Festkörperphysik, Technical University Darmstadt, 64289 Darmstadt, Germany
}

(May 18, 1999)

\begin{abstract}
The magnetic properties of the two-channel periodic Anderson model for uranium ions, comprised of a quadrupolar and a magnetic doublet are investigated through the crossover from the mixedvalent to the stable moment regime using dynamical mean field theory. In the mixed-valent regime ferromagnetism is found for low carrier concentration on a hyper-cubic lattice. The Kondo regime is governed by band magnetism with small effective moments and an ordering vector $q$ close to the perfect nesting vector. In the stable moment regime nearest neighbour anti-ferromagnetism dominates for less than half band filling and a spin density wave transition for larger than half filling. $T_{m}$ is governed by the renormalized RKKY energy scale $\mu_{\text {eff }}^{2}<X_{\sigma}>^{2} J^{2} \rho_{0}(\mu)$.
\end{abstract}

Introduction. Heavy Fermion (HF) materials [1] have drawn much attention since the discovery of superconductivity in $\mathrm{CeCu}_{2} \mathrm{Si}_{2}$ [2] which is characterized by an anisotropic order parameter with symmetry yet to be determined. Over the years, it became clear that the heavy Fermi-liquid is not the ground state: almost all HF materials show magnetic or superconducting phase transitions, which either compete with each other in Ce based compounds or coexist in Uranium based materials. Additionally, doubts were casted on whether the Fermi-liquid picture is even applicable for the normal state properties in some U-based [3] or doped compounds [4].

In this paper, new results on magnetism in the twochannel periodic Anderson model (TCPAM) [5] are presented. In the TCPAM, the restricted local Hilbert space, consisting of a quadrupolar and a magnetic ground state doublet for two different valence configurations, models an Uranium ion in a cubic environment, which can either fluctuate between $5 f^{2} / 5 f^{3}$ or $5 f^{1} / 5 f^{2}$ ground state configurations. On symmetry grounds, the coupling of the ion to the itinerant electrons via hybridization can be characterized by an orbital and a spin label generating an effective two band model [3].

It has been shown that the paramagnetic phase exhibits non-Fermi-liquid properties, as manifested in a large residual resistivity [5]. They are caused by the overcompensated effective impurity on each Uranium site acting as incoherent scatterer. The predicted absence of a Drude peak in the optical conductivity [5] has been found experimentally in $\mathrm{UBe}_{13}$ [6]. On a lattice, however, the residual entropy [7] is expected to be quenched by a phase transition. Candidates are magnetic, channel ordering or superconducting transitions, which all have to bind the residual magnetic or quadrupolar spin. In the periodic two-channel Kondo model, anti-ferromagnetic and charge ordering phase transitions have been reported in the strong coupling limit of the model $|J| \rho_{0}(0)>0.5$ [8], $\rho_{0}(\epsilon)$ being the non-interacting density of states. Additionally, the authors interpret a sign change in the pair susceptibility for a spin/channel singlet sector as a superconducting transition. The absence of a divergence is viewed as indicator of a first order transition.

In this paper, I focus on possible spin transitions. I have calculated the spin phase transition temperature $T_{m}$ as a function of band filling and energy difference $\epsilon_{f}=E_{\sigma}-E_{\alpha}$ between the quadrupolar and the magnetic doublet using the dynamical mean field theory [9]. Varying $\epsilon_{f}$ continuously allows me to investigate three different regimes: $\left|\epsilon_{f}\right|$ smaller than the charge fluctuation energy $\Delta$ defines the intermediate valence regime, which has recently been discussed experimentally [10,11] and theoretically 12,13] as a possible scenario for $\mathrm{U}_{1-x} \mathrm{Th}_{x} \mathrm{Be}_{13}$. The local moments are not well defined, and the carriers rather mobile. Magnetic ordering would have to involve a gain in kinetic energy since the effective exchange couplings are small [14]. Ferromagnetism is found at low carrier concentration. For $\left|\epsilon_{f} / \Delta\right| \approx 2$, the Kondo effect dominates. Band magnetism with very small effective magnetic moments is expected since all available electrons are involved in the screening process. For large $\left|\epsilon_{f} / \Delta\right|$, magnetism is governed by stable local moments whose interactions are mediated by the conduction electrons (RKKY interaction).

Theory. The two channel Anderson lattice Hamiltonian under investigation reads

$$
\begin{aligned}
\hat{H}= & \sum_{\alpha<i, j>} \frac{t^{*}}{\sqrt{d}} c_{i \alpha \sigma}^{\dagger} c_{j \alpha \sigma}+\sum_{i \sigma} E_{\sigma} X_{\sigma, \sigma}^{(i)}+\sum_{i \alpha} E_{\alpha} X_{\alpha, \alpha}^{(i)} \\
& +\sum_{i \sigma \alpha} V\left\{c_{i \alpha \sigma}^{\dagger} X_{-\alpha, \sigma}^{(i)}+h . c\right\} .
\end{aligned}
$$

$X$ are the Hubbard operators, $d$ being the spatial dimension, and $t^{*}$ the reduced hopping matrix element of the conduction electrons between nearest neighbours. The $c$ electrons are created by $c_{i \alpha \sigma}^{\dagger}$ at the lattice site $i$, labeled 
by a spin $\sigma$ and a channel index $\alpha= \pm$ and couple via the hybridization matrix element $V$ to the ionic manybody states on each lattice site. We will assume that the energies of the magnetic doublets split linearly under an applied small external field, and van Vleck contributions are neglected.

In the limit of infinite spatial dimensions the two particle-hole irreducible vertex $\Gamma_{f f}$ becomes local [15]. Using the dynamical mean field theory (DMFT) [9] in finite dimensions, the Bethe-Salpeter equation for the full two particle-hole propagator in f-electrons reads

$$
\underline{\underline{\Pi}}_{f f}\left(i \nu_{n}\right)=\beta \underline{\underline{\chi}}_{f f}^{0}+\underline{\underline{\chi}}_{f f}^{0} \frac{1}{\beta} \underline{\underline{\Gamma}}_{f f} \underline{\underline{\Pi}}_{f f}\left(i \nu_{n}\right),
$$

where $\chi_{f f}^{0}$ is the free particle-hole $f f$-propagator

$$
\begin{aligned}
& \chi_{f f}^{0}\left(i \omega_{n}, i \omega_{m} ; i \nu_{n}, \underline{q}\right)=-\frac{\delta_{n, m}}{N} \sum_{\underline{k}} F_{\underline{k}}\left(i \omega_{n}+i \nu_{n}\right) F_{\underline{k}+\underline{q}}\left(i \omega_{n}\right) \\
& =\int d \epsilon_{1} d \epsilon_{2} \rho_{2}^{0}\left(\epsilon_{1}, \epsilon_{2}, \underline{q}\right) F\left(\epsilon_{1}, i \omega_{n}+i \nu_{n}\right) F\left(\epsilon_{2}, i \omega_{n}\right)
\end{aligned}
$$

and $F_{\underline{k}}(z)=F(\epsilon(\underline{k}), z)$ is the lattice $f$-Green's function. The matrices are indexed by Matsubara frequencies, $\Pi_{f f}\left(i \omega_{n}, i \omega_{m} ; i \nu_{n}\right)=\left.\underline{\underline{\Pi}}_{f f}\right|_{i \omega_{n}, i \omega_{m}}$, and $\rho_{2}^{0}\left(\epsilon_{1}, \epsilon_{2}, \underline{q}\right)$ is the two particle density of states given by the underlying lattice [16]. Since Eqn. (2) also holds for the local two-particle propagator $\underline{\Pi}_{f f}^{l o c}$, we obtain the irreducible vertex $\Gamma_{f f}$ and substitute it into (2) to derive

$\underline{\underline{\Pi}}_{f f}(\underline{q})=\underline{\underline{\Pi}}_{f f}^{l o c}\left[\underline{\underline{1}}-\frac{1}{\beta}\left[\left[\underline{\underline{\chi}}_{f f}^{0}\right]_{l o c}^{-1}-\left[\underline{\chi}_{f f}^{0}(\underline{q})\right]^{-1}\right] \underline{\underline{\Pi}}_{f f}^{l o c}\right]^{-1}$

which is valid for each Boson frequency $i \nu_{n}$ separately 21,22. In order to study possible phase transitions, I restricted my calculations to the fully symmetric phase. I used a Gaussian non-interacting density of states $\rho_{0}(\epsilon)=$ $\frac{1}{t^{*} \sqrt{\pi}} e^{-\left(\epsilon / t^{*}\right)^{2}}$ for a simple cubic lattice in large dimensions, and focused on the static $f f$-spin susceptibility

$$
\chi_{f f}(\underline{q})=\frac{1}{\beta^{2}} \sum_{i \omega_{n}, i \omega_{m}} \Pi_{f f}\left(i \omega_{n}, i \omega_{m} ; 0, \underline{q}\right) e^{\delta\left(i \omega_{n}+i \omega_{m}\right)} .
$$

The diverges of $\chi_{f f}(\underline{q})$ originating from zeros of the denominator of (4) determine the phase transitions, resembling a Stoner like expression for the lattice susceptibility. I would like to mention that the convergence factors in Eqn.(5) are important for evaluating the susceptibility analytically and numerically. The conduction electron susceptibility $\chi_{c c}$ and the mixed terms $\chi_{c f}$ and $\chi_{f c}$ also contribute to the total susceptibility. However, since the unperturbed conduction electrons form a Fermi-gas, any irreducible interaction is generated by the $f$-electrons [20]. Therefore, divergences occur collectively for all contributions when $\chi_{f f}(\underline{q})$ diverges due to $c-f$ mixing.
In large spatial dimensions, $\rho_{2}^{0}\left(\epsilon_{1}, \epsilon_{2}, \underline{q}\right)$ obtains its $q$ dependence only through lattice dependent structure factor $\eta(\underline{q})=\sum_{i} \cos \left(q_{i}\right) / D$ in the case of a hyper cubic lattice of $D$-dimensions 16$]$.

In context of Heavy-Fermion systems [1], the terms anti-ferromagnetism (AF) and spin density wave (SDW) are often used interchangeably, since it is hard to distinguish experimentally true incommensurable band magnetism from complicated yet commensurable magnetic structures. Here, I will call a transition antiferromagnetic if the divergence occurs at $\eta(\underline{q})=-1$, ferromagnetic, if $\eta(\underline{q})=1$, while all other cases will be labeled as $S D W$. To be specific, I choose the wave vector $\underline{q}=q_{0}(1,1, \cdots)$ along the nesting direction instead of $\eta(\underline{q})^{-}$in all figures. I define the characteristic temperature of the lattice $T^{*}$ as the temperature at which the initial moment of the lower lying doublet is screened down to $40 \%$ [12]. This energy scale is the lattice analog to the single ion Kondo temperature. Taking into account the lack of universality due to the ongoing renormalization of the conduction band for $T<T^{*}$, it is not surprising if $T^{*}$ does not coincide with the energy scale of the electrical response $T_{F L}$, as demonstrated in Ref. [17]. Hints are also seen in calculations for low carrier concentrations [18.

Results. The Anderson width $\Delta=V^{2} \pi \rho_{0}(0)$ is used throughout the paper as the energy unit. All calculations have been performed for a fixed band width $t^{*}=10$ which yields the hybridization strength $V^{2}=\Delta t^{*} / \sqrt{\pi}$. If one defines the effective dimensionless coupling constant $g=\rho_{0}(0)|J|=\rho_{0}(0) V^{2} /\left|\epsilon_{f}\right|=\Delta /\left(\pi\left|\epsilon_{f}\right|\right)$, and requires, that $\Delta / \epsilon_{f} \mid<1$ as a criterion for excluding the intermediate valence regime, the TCPAM can only describe the weak coupling regime $(g<1 / \pi)$ of the two-channel Kondo lattice model. The chemical potential $\mu$ has been varied from -5 to 5 to obtain different conduction electron occupancies $n_{c}$ : at $n_{c}=4$ the c-bands are completely filled.

The occupancy of the magnetic doublet decreases monotonically with increasing $c$-band filling. Even though the effective DOS is altered symmetrically around half filling, the different $f$-valent states $|\sigma\rangle(\mid \alpha>)$ involve admixture of virtual hole (particle) band states which are increased (decreased) with decreasing chemical potential. Therefore, $T^{*}$ decreases more rapidly with conduction band depletion than with conduction band filling. Moreover, assuming $T^{*} \propto \exp -1 /(J \rho)$, the change of $T^{*}$ becomes more significant for decreasing $J$. This is exactly what is found numerically of the occupancy dependence of $T^{*}\left(n_{c}\right)$ for different $\epsilon_{f}$.

Generally, magnetic phase transitions in the periodic Anderson model are expected to be AF for the stable moment or SDW for the Kondo regime [1] stemming from inter and intra band excitations in a hybridized band picture. This picture is not truly applicable in the two channel model due to lack of quasi-particles at the chem- 




FIG. 1. Mixed-valent regime: $T_{m} / T^{*}$ versus $q_{0}$ for different conduction electron fillings. A ferromagnetic transition is found from $1 / 4$ to $1 / 2$ conduction band filling for $\epsilon_{f}=-1 \Delta$. The inset shows $T_{m} / T^{*}$ for $\epsilon_{f}=0$.

ical potential.

In the mixed valent (IV) regime $\left(\epsilon_{f} / \Delta=-1\right)$ the largest $T_{m}$ is found for a ferromagnetic transition at less than half band filling (Fig. 1). With the exception of $n_{c}=1, T_{m} / T^{*}<1$. Independently, I also verified the existence of a ferromagnetic phase by calculating the spin polarization in an external magnetic field: its magnitude was decreased from a finite value of the order of $10^{-4} T^{*}$ to zero field. A zero field magnetization was found only below $T_{m}$. Since the used XNCA method works reliably only in the multi-channel case 5], accurate determination of a magnetization curve below $T_{m}$ was not possible.

In the single channel PAM, no magnetism is expected in the IV regime due weakness of the effective exchange couplings. In the two channel PAM, however, incoherent scattering of the conduction electrons remains present in the paramagnetic phase down to low temperatures. Energy can be gained by reducing the scattering rate in a spin-polarized state. This gain grows with increasing occupancy of the magnetic doublet. Therefore, the transition is found at low $c$-carrier concentration, where charge fluctuations mixes increasing number of $c$-hole states to local magnetic ground state, stabilizing the local moment. Because charge fluctuations are ignored in the Kondo lattice model, it has not been observed before [8]. The FM is clearly a strong coupling effect, since it is caused by high energy charge fluctuations [19]. It leads to a higher mobility of the $c$-electrons measurable through an enhancement of DOS in the majority spin above the chemical potential. In the FM phase, the increasing magnetization quenches the spin channel and the model crosses over to a single channel PAM [5]. Below $T_{m}$, Fermi-liquid properties are regained.

The picture changes significantly in the crossover

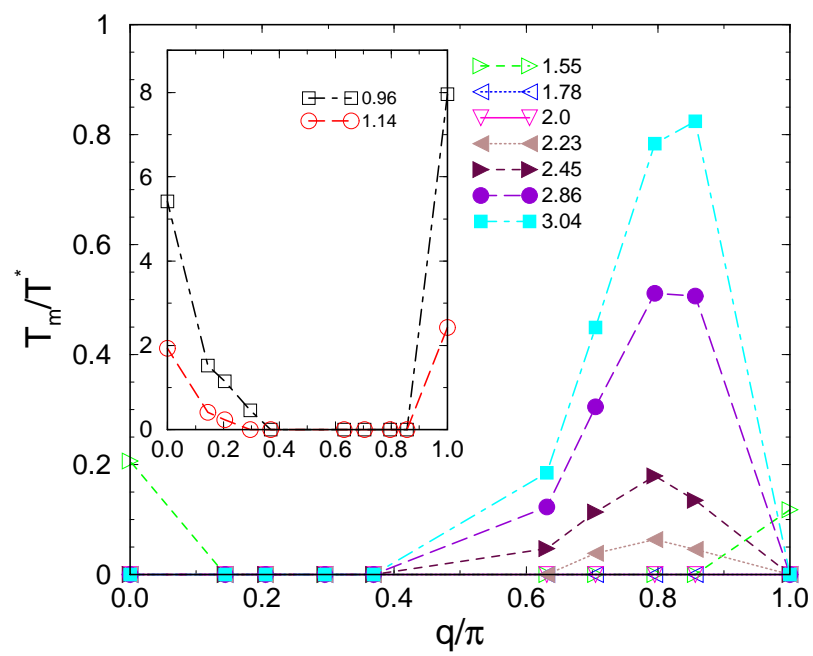

FIG. 2. Kondo regime: $T_{m} / T^{*}$ versus $q_{0}$ for different conduction electron fillings. $\epsilon_{f}=-2 \Delta$. The inset shows $T_{m} / T^{*}$ near quarter filling.

regime, $\epsilon_{f} / \Delta=-2$ (Fig. 2): although the occupancy changes moderately from 0.82 to 0.74 with increasing band filling, the maximum of $T_{m}$ is now found at a finite $q$ vector $q_{0} \approx .85 \pi$ indicating a SDW transitions for band fillings larger than 0.5. Around half filling, the Kondo effect dominates. The effective local moment is already quenched to $7 \%$ of its high temperature value at the SDW transition temperature $T_{m}$. The transition occurs well below $T^{*}$ while with increasing effective moment, the transition is shifted towards higher temperatures. Near quarter filling, the local moments are only weakly screened: the effective moment $\mu_{\text {eff }}^{2}=0.6 \mu_{\text {free }}^{2}$, the ratio $T_{m} / T^{*}$ is very large (inset of Fig. 2) and an $\mathrm{AF}$ transition is observed similar to the stable moment situation discussed next.



FIG. 3. Stable moment regime: $T_{m} / \Delta$ versus $q_{0}$ for different conduction electron fillings. $\epsilon_{f}=-3.5 \Delta$.

Lowering $\epsilon_{f}$ further, we arrive at the stable local mo- 
ment regime (Fig. 3). For $n_{c}<2$, an AF transition is found. Note that $T_{m}$ is not rescaled with $T^{*}$ in this case, indicating that the screening of the moments is very low (about 25\%). Increasing the band filling, the admixture of $\mid \alpha>$ states into the ground state increases, which decreases the local moments: for $n_{c}>2$ SDW transitions at large $q$ vectors are found. For positive $\epsilon_{f}$, no magnetic phase transition is found.

Discussion. I have established, for the first time, a ferromagnetic solution of the two-channel periodic Anderson model in the intermediate valence regime for low carrier concentration. Energy and mobility is gained by reducing the incoherent scattering of the conduction electrons in a spin-polarized state. The loss of energy due to spin polarization of the conduction band is low for small band fillings. The transition is driven by high energy charge fluctuations indicating the strong coupling nature of the FM transition. The presences of FM transitions and strong ferromagnetic fluctuations suggest the possibility of triplet superconductivity. Preliminary studies indicate a divergence of the pair susceptibility in the spin/channel triplet sector, implying an odd-frequency gap function.

In the Kondo regime, I find SDW transitions, with a large wave vector $q$, for band fillings larger than $1 / 2$. Additionally, nearest neighbour AF and tendency towards ferromagnetism is observed for low carrier concentrations. In the stable moment regime AF transitions are found at less than half filling and SDW transition above half filling. However, I have excluded a detailed treatment of band-structure effects in finite dimensions within DMFT, which can be included through Equ. (3). The bare two-particle density of states then becomes truly $\underline{q}$-dependent. I hope to clarify its influence on the spin susceptibility in three dimensions in the near future.

In all the cases I investigated, I found $T_{m} \propto E_{R K K Y}=$ $\mu_{e f f}^{2}\left\langle X_{\sigma}\right\rangle^{2} J^{2} \rho_{0}(\mu)$. where $\left\langle X_{\sigma}\right\rangle$ is the occupation number of the magnetic ground state doublet of the each ion. The ratio $E_{R K K Y} / T_{m}$ decreases monotonically from around 3. to 1.5 with decreasing effective local moment for $\epsilon_{f}=-2$. The magnetic transition is always governed by an effective RKKY energy $E_{R K K Y}$, which takes into account the screening of the local moments. $E_{R K K Y}$ can be one order of magnitude smaller than the bare RKKY energy.

Experimentally, the change of parameters in real materials is much more difficult: usually either the size of the unit cell is changed through isostructural substitution or through external pressure. Essentially, the overlap between nearest neighbours is varied which yields a change in the hybridization $V$ and the bandwidth $t^{*}$. It is believed, that the core energy difference $\epsilon_{f}$ stays unaltered [23. Under the assumption, that only hybridization and bandwidth are altered by pressure, application of positive pressure will be equivalent to a reduction of $\left|\epsilon_{f}\right|$. The results presented show clearly the same tendency as observed in experiments 17: the application of pressure leads to a suppression of magnetic phase transitions. Local moments are destroyed through hybridization induced de-localization of the f-electrons.

The author is very thankful for fruitful discussions with Dr. Th. Pruschke and Prof. N. Grewe, who also carefully read the manuscript. The work was in part funded by the Sonderforschungsbereich 252, Elektronisch hochkorrelierte metallische Materialien. All calculation have been performed on a 8 CPU LINUX cluster.

[1] For a review see: N. Grewe and F. Steglich, in Handbook on the Physics and Chemistry of Rare Earths, edited by K. A. Gschneidner, Jr. and L. Eyring (Elsevier, Amsterdam, 1991), Vol. 14, p. 343.

[2] Steglich et. al., Phys. Rev. Lett.43, 1892 (1979).

[3] D. L. Cox, Phys. Rev. Lett. 59, 1240 (1987).

[4] For on overview see: M.B. Maple et. al. , J. Low Temp. Phys. 99, 223 (1995).

[5] F. B. Anders, M. Jarrell and D. Cox, Phys. Rev. Lett. 78, 2000 (1997).

[6] F. Bommeli et. al , Phys. Rev. B 56, R10001, (1997).

[7] for a review on the two channel Kondo impurity physics see: D. L. Cox, A. Zawadowski Advances in Physics, 47, 599 (1998).

[8] M. Jarrell, H. Pang, D.L. Cox, Phys. Rev. Lett 78, 1996 (1997)

[9] For a review, see A. Georges, G. Kotliar, W. Krauth and M. J. Rozenberg, Rev. Mod. Phys. 68, 13 (1996).

[10] F. Aliev et. al, Europhys. Lett. 32, 765 (1995).

[11] F. Aliev et. al, J. Phys.: Condens Matter 8, 9807 (1996).

[12] A. Schiller, F. B. Anders, D. L. Cox, Phys. Rev. Lett. 81, 3235 (1998).

[13] M. Koga, D. L. Cox, Phys. Rev. Lett. 82, 2575 (1999)

[14] N. Grewe, H. Keiter, Phys. Rev. B 24, 4420 (1981).

[15] U. Brand, Chr. Mielsch, Z. Phys. B 75, 365 (1989).

[16] E. Müller-Hartmann, Z. Phys. B. 74, 507 (1989), 76, 211 (1989).

[17] S. Süllow, M. C. Aronson, B. D. Rainford, and P. Haen, Phys. Rev. Lett. 82, 2963 (1999).

[18] A. N. Tahvildar-Zadeh, M. Jarrell, J. K. Freericks condmat/9610188

[19] Since the mechnism appears to be generic, it would be worthwhile to investigate, whether the FM transitions in CeSb can be understand along the same lines.

[20] N. Grewe, Z. Phys. B. 67, 323 (1987).

[21] M. Jarrell, Phys. Rev. B51, 7429 (1995).

[22] Th. Pruschke,Th. Obermeier, J. Keller, J. Phys.: Condens. Matter 8, 3161 (1996).

[23] J. Röhler, in Handbook on the Physics and Chemistry of the Rare Earths, edited by K. A. Gschndner, L. R. Eyring, and S. Hüffner (North Holland, Amsterdam, 1987), Vol 10, p492. 